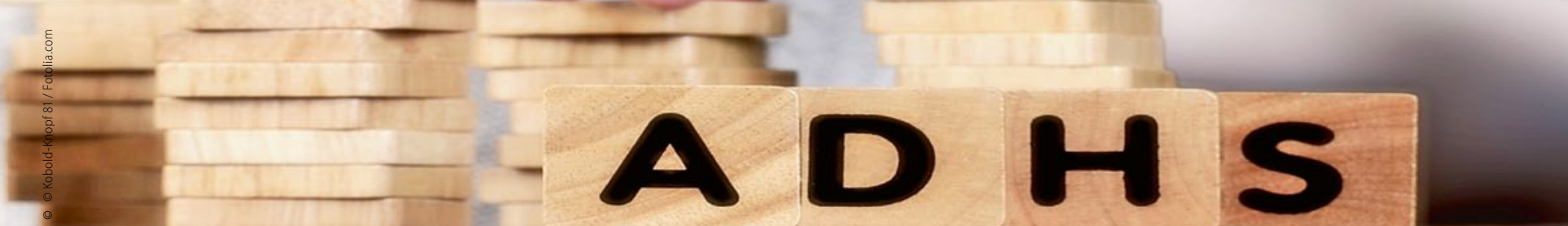

\title{
Was ist dran an den „Überdiagnosen“?
}

Eine ADHS-Diagnoserate von $18 \%$ bei Elfjährigen und Verordnungszahlen von $13 \%$ bei Zwölfjährigen in einzelnen Regionen Deutschlands lassen aufhorchen. Diese Zahlen brachte der im Januar 2013 veröffentlichte BARMER-GEK-Arztreport zutage. Zahlreiche - zum Teil polemische - Artikel auch in renommierten Zeitungen und Zeitschriften waren die Folge. Nachdem nun wieder andere emotionsbesetzte Themen wie Pferdefleisch im Essen oder die Eurokrise auf Zypern die Titelseiten der Tageszeitungen besetzen und es um das Thema ADHS ruhiger wurde, lohnt sich ein nochmaliger Blick auf den Report.

Demnach wird ADHS in Deutschland am häufigsten bei Jungen im vierten Schuljahr in der ambulanten Versorgung diagnostiziert (11,9\%), das heißt, jeder achte Junge des Geburtsjahrs 2001 erhielt 2011 die Diagnose „hyperkinetische Störung“. Insgesamt liegt die Diagnoserate damit bei Jungen dreimal höher als bei Mädchen. Während es einen eventuellen Trend - insbesondere in einigen Regionen Deutschlands - zu einer sehr häufigen Diagnose ADHS zu geben scheint, fällt die administrative Prävalenz in Adoleszenz und jungem Erwachsenenalter deutlich ab. In der Altersgruppe der 20- bis 24-Jährigen liegt die Diagnoserate laut Report bei $0,8 \%$, bei über 25-Jährigen bei $0,3 \%$. Im Fünfjahresvergleich ist zwar auch hier ein deutlicher Anstieg um den Faktor 3 zu sehen. Im Vergleich zur erwarteten Prävalenz von $2 \%$ deuten die Zahlen in diesen Altersgruppen jedoch nicht auf eine gegenwärtige Überdiagnose hin, sondern dürfen vielmehr als Ausdruck einer inzwischen zur Kenntnis genommenen möglichen Persistenz der ADHS in das Erwachsenenalter aufgefasst werden, welche in Deutschland lange Zeit weit unterschätzt wurde. Die Verordnungszahlen zeichnen ein ähnliches Bild: In der Altersgruppe der Zehn- bis Zwölfährigen werden am häufigsten Stimulanzien und andere bei ADHS zugelassene Medikamente verordnet. Im Jugendalter findet sich wiederum ein deutlicher Abfall. Im Erwachsenenalter erhalten noch 0,2\% ADHS-spezifische Medikation. Kontakte zu psycho-

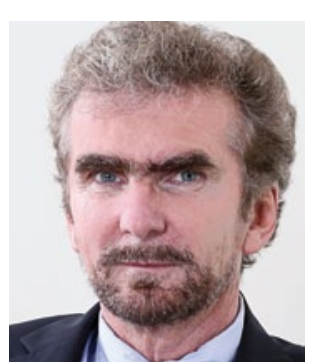

Prof. Dr. med. Mathias Berger

Ärztlicher Direktor der Abteilung für

Psychiatrie und Psychotherapie

Universitätsklinikum Freiburg i. Br.

logischen Psychotherapeuten (nur diese wurden erfasst) wurden bei knapp $10 \%$ der Kinder und Jugendlichen dokumentiert, zu Erwachsenen liegen dazu keine Daten vor.

Was sagen uns nun diese Zahlen? Zum einen scheint es insbesondere in einzelnen Regionen Deutschlands um das vierte Schuljahr herum, also zur Zeit der Weichenstellung in weiterführende Schulen, einen Trend zu einer möglichen „Überdiagnose“ sowie „Übermedikation“ zu geben, der nochmals die Wichtigkeit leitliniengestützter Diagnostik und Therapieempfehlungen verdeutlicht. Zum anderen scheinen ältere Jugendliche und junge Erwachsene noch nicht ausreichend diagnostisch wahrgenommen und damit auch nicht adäquat medikamentös und psychotherapeutisch behandelt zu werden. Dies ist insofern kritisch, als dass die Persistenz der ADHS in das Erwachsenenalter mit zahlreichen psychosozialen Einschränkungen und weiteren psychischen Störungen einhergehen kann. Ziel sollte es daher sein, entsprechende auf Transition ausgerichtete Versorgungsmodelle zu etablieren und wissenschaftlich zu evaluieren, die sowohl medikamentöse als auch psychotherapeutische Behandlungsoptionen umfassen.

Mathias Berger 